\title{
GRAPHIC: \\ The Geneva Reduction and Analysis Pipeline for High-contrast Imaging of planetary Companions
}

\section{Janis Hagelberg, Damien Ségransan, Stéphane Udry, and François Wildi}

Observatoire de Genève, University of Geneva, 51 Chemin des Maillettes, 1290, Versoix, Switzerland email: janis.hagelberg@unige.ch

\begin{abstract}
We present a new analysis and reduction pipeline for the detection of planetary companions using Angular Differential Imaging. The pipeline uses Fourier transforms for image shifting and rotation in order to achieve very low signal loss. Furthermore it is parallelised in order to run on computer clusters of up to 1024 cores. The pipeline was developed in Geneva for the ongoing direct imaging campaign for stars with radial velocity drifts in the HARPS and CORALIE radial-velocity planet-search surveys. In addition to that, a disk mode has been implemented in the context of observations of the protoplanetary disk around HD142527.
\end{abstract}

Keywords. instrumentation: adaptive optics, techniques: high angular resolution, techniques: image processing, methods: data analysis, planetary systems, stars: low-mass, brown dwarfs

\section{Introduction}

Direct imaging techniques based on Angular Differential Imaging (ADI) (Marois et al. 2006) which use field rotation in order to subtract the stellar point-spread-function, are currently the techniques achieving the highest contrast detections at small separations. ADI relies on shifts of well aligned frames and image derotation to correct for the field rotation. We developed a pipeline called GRAPHIC (Hagelberg et al. in prep) based on Fourier transforms to achieve high fidelity image transformations in order to push detections limits of ADI even further.

\section{ADI using Fourier transforms}

The main advantage of Fourier-based image processing over the conventional direct interpolation based methods is that interpolation is done on spatial frequencies. As a result the original signal isn't changed and only some additional very high frequency noise gets injected: conversely spline interpolation algorithms act as low-pass filters spreading out the noise and putative companions signal.

The two main hurdles to overcome in order to use Fourier-based image processing is on one hand the additional computation time linked to the intensive use of FFTs and on the other hand the Gibbs oscillations caused by sharp image features such as bad pixels or image borders. The processing time can drastically be reduced thanks to massive parallelisation, however removing sharp image features whitout loosing signal needs extra care.

Using the translation property of Fourier transforms, we shift each frame by applying a two-dimensional phasor. This way, to recenter a frame by a sub-pixel step $a$ in $x$ and $b$ in $y$, we apply a phasor of the form $e^{-i 2 \pi\left(\nu_{x} \cdot a+\nu_{y} \cdot b\right)}$ to the Fourier transformed image. 


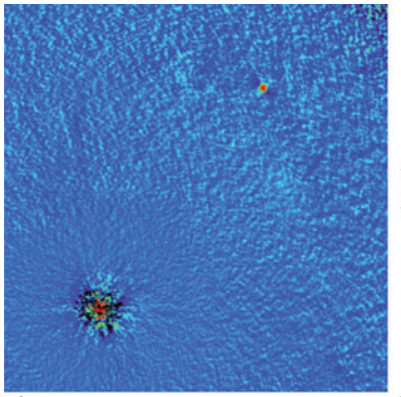

a)

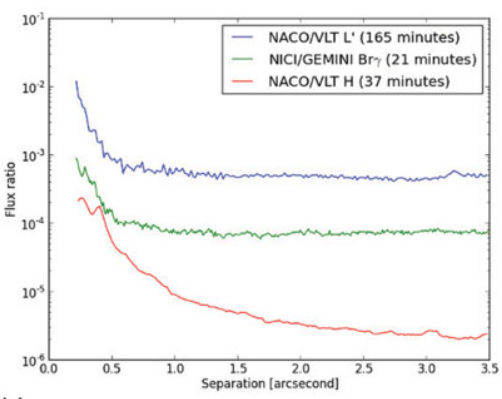

b)

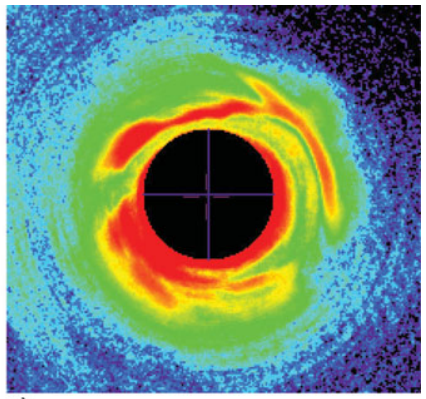

C)

Figure 1. Applications of GRAPHIC: a) Direct detection of companion candidates revealed by radial velocity trends in the HARPS and CORALIE data. b) $5 \sigma$ detection limits for various instruments, filters, and total integration time combinations. c) NICI/GEMINI Ks band imaging of the protoplanetary disk around HD142527 reduced using the prototype GRAPHIC pipeline Casassus et al. (2013).

The Fourier-based rotation is implemented using the three shears algorithm described by Larkin et al. (1997) and Unser et al. (1995). It is based on the property that a rotation matrix can be decomposed into a product of three shear matrices.

\section{Applications of GRAPHIC}

The pipeline was primarily developed to reduce data from the ongoing L' band ADI campaign on NACO/VLT for stars with radial velocity drifts in the HARPS and CORALIE radial-velocity planet-search surveys.

GRAPHIC was also applied to some data from the coordinated European Large Program using $\mathrm{H}$ band ADI with NACO which aims to provide a comprehensive and statistically significant study of the occurrence of extrasolar giant planets and brown dwarfs at large (5-500 AU) orbital separation around 150 young, nearby stars (Zurlo et al. 2013).

The previous version of the pipeline (PADIP) was used to reduce NICI/GEMINI data of the protoplanetary disk around HD142527, and to search for companions within the protoplanetary gap (Casassus et al. 2013). In this framework a disk mode has been developed to minimise the self-subtraction artefacts which tend to appear when using ADI for disk imaging (Milli et al. 2012). Performance comparison with other pipeline and further details on the disk mode are given in Casassus et al. 2013.

\section{References}

Casassus, S., Plas, G. van der, M, S. P., Dent, W. R.F., Fomalont, E., Hagelberg, J., Hales, A., Jordán, A., Mawet, D., Ménard, F., Wootten, A., Wilner, D., Hughes, A. M., Schreiber, M. R., Girard, J. H., Ercolano, B., Canovas, H., Román, P. E., \& Salinas, V. 2013, Nature, 493, 191

Hagelberg J., Ségransan D., Udry S., Wildi F., in prep.

Larkin, K. G., Oldfield, M. A., \& Klemm, H. 1997, Opt. Commun., 139, 99

Marois, C., Lafrenière, D., Doyon, R., Macintosh, B., \& Nadeau, D., 2006 ApJ, 641, 556

Milli, J., Mouillet, D., Lagrange, A.-M., Boccaletti, A., Mawet, D., Chauvin, G., Bonnefoy, M. 2012, A\& A, 545, 111

Unser, M., Thevenaz, P., Yaroslavsky, L. , 1995 IEEE IP, 4, 1371

Zurlo, A., Vigan, A., Hagelberg, J., Desidera, S., Chauvin, G., Almenara, J. M., Biazzo, K., Bonnefoy, M., Carson, J. C., Covino, E., Delorme, P., D’ Orazi, V., Gratton, R., Mesa, D., Messina, S., Moutou, C., Ségransan, D., Turatto, M., Udry, S., \& Wildi, F., 2013 A\& A, 554,21 